\title{
Patterned Endorsement Framework for Defense Sector using Two Level Voice Authentication Process
}

\author{
M.Kathiresh ,N.Shanmuganathan,R.Sankarasubramanian
}

\begin{abstract}
The guard segment considers security usage as the essential and critical errand to be executed so usage ought to be made with more concern. One of the ideal arrangements is Voice for upgrading the security framework in which the confirmation of clients is performed with the guide of their voice signals. This confirmation has been performed by actualizing Security Voice Authentication System (SVAS) strategy in our past work. Be that as it may, this philosophy isn't a lot of viable on the insider aggressors. The security framework would be separated by the vindictive/narrow-minded clients as they go about as genuine clients by transmitting the voice sign of unique clients. This deficiency can be overwhelmed by presenting a procedure called Two Level Voice Authentication System (TLVAS). This examination includes producing mystery keys at first by methods for arbitrary qualities inputted by the client. It will give security in the two-level procedure which incorporates the respectability of the handling ought to be solid. The sign must be changed over which control the figuring techniques. This strategy is seen as increasingly powerful against the insider assailants by avoiding the Compromisation created by them. Installing procedure used to execute for doing the exhibition investigation and the examination is completed by methods for expanded security level.
\end{abstract}

Keywords: Voice authentication, Compromisation attack, two-level voice process, compression, random secret key generation

\section{INTRODUCTION}

Biometric confirmation, when all is said in done, utilizes the novel natural attributes which are not the same as passwords and token-based verification for checking the character of an individual [1]. This validation is hard for re-making and it wipes out the requirement for recollecting passwords or conveying it in person joined by the risk of misfortune or being taken [2]. This is progressively beneficial to the clients as it is a piece of a person. Voice acknowledgment (otherwise called voice confirmation or speaker acknowledgment) helps in verifying the people's personality by methods for examining the voice of an individual [3]. An exceptional "voiceprint" is picked up by voice designs that are gotten by methods for development and state of mouth and jaw, aviation routes and delicate tissue pits [4].

Revised Manuscript Received on November 15, 2019

M.Kathiresh, Research Scholar, Department of Computer Science, Erode Arts and Science College, Erode, Email: kathireshcs83@gmail.com

N.Shanmuganathan, Research Scholar, Department of Computer

Science, Erode Arts and Science College, Erode, rahulntr105@gmail.com

Dr.R.Sankarasubramanian, Associate Professor, Department of

Computer Science, Erode Arts and Science College, Erode,

rsankarprofessor@gmail.com
Speaker acknowledgment falls under a sort of voice acknowledgment innovation [5]. Be that as it may, this innovation is very surprising from the other remote helpers and discourse to-content applications likely Alexa or Siri [6]. Discourse acknowledgment has an incredible detriment, that however the verbal language can be perceived by it, it doesn't recognize the speaker relying upon the novel vocal traits. This deficiency could be overwhelmed by the biometrics. Sans's hand's versatile validation is voice confirmation's as a matter of first importance utilization [7]. This kind of approval is more pleasant and ideal for customers than various settings likely one of a kind finger impression affirmation [8], facial affirmation and various sorts of biometric checks which might be a burden on the vehicles [9].

Voice confirmation likewise helps in discourse acknowledgment gadgets to be specific to Amazon's Alexa and Google Home [10]. Menial helpers are generally utilized for putting requests and performing different capacities which thusly requests some type of verification. During the client assistance calls, speaker acknowledgment goes about as an authenticator that focuses [11]. This is seen as progressively secure and advantageous by clients as this framework dispensed with them from sharing their data likely the charge card number or permit for checking their character.

To separate the various speakers, the voice check framework assembles the different attributes of voice with the end goal that the sort of voice is separated univocally for recognizing the individual who is talking. "The physical state of the vocal tract" is identified with the physiology viewpoints [12]. This is affected by the development of larynx, jaw, tongue, pharynx, lungs, and mouth. The distinction in the voice can be watched dependent on the sound delivered by the development of the air, as those components are seen as various structures in each person. Every single diverse viewpoint likely habits and developments choose the conduct perspectives which extraordinarily impact the discourse [13]. Those investigations significantly help in the distinguishing proof of the voice of a person by preparing the verbally expressed sentence in the methods for portioning the sentence into divisions and changing over those fragments into a computerized group. All the exceptional vocal attributes, for example, pitch, tone, and rhythm are considered while breaking down each portion.

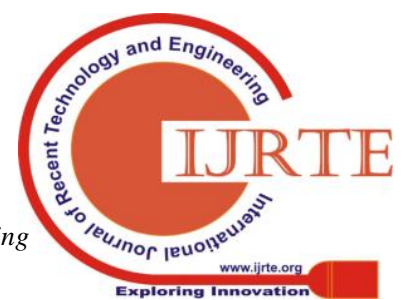


To decide the nature of the voice test and correlation of voice among the person to be performed, later on, redundancy of an expression or a succession number is required by the client, with the end goal that the example will be acknowledged and bolstered into the database. This likewise guarantees when an individual talks the approval sentence, the info is being broke down and contrasted with the database with discovering whether the pieces of information are available in the database by a similar person. Various frameworks appeared for the validation of an individual's voice. They are named subject to the content and autonomous of content

The content ward verification framework requests the client to enter the foreordained sentence to affirm his confirmation [14]. This stage is very known as "passphrase". The content autonomous framework, then again, isn't subject to the passphrase. It requests the client to articulate the content very long. In this structure, there is certifiably not a solitary bond knowledge into the substance of the speaker for his check. Therefore, this makes a probability for appraisal and assessment of voices in a wide scope of points of view that makes it unique.

This work chiefly goes for the usage of the voice validation framework to guarantee the foundation of a verified situation in the guard segment. This exploration makes the productive validation of voice signals for guaranteeing the safe condition in the resistance segments. A superior result is gotten from this investigation in correlation with the current techniques.

\section{RELATED WORKS}

The creator M. Shamim Hossain et al. depicted a cloud-helped discourse and face acknowledgment structure for the human services framework. The patient's Condition assembled from their discourse and face pictures. Further arranged by the help vector machine. At long last, it advances to the remote consideration focus, from that the experts give the best possible answer for the patients [5].

The creator Qian Wang et al. Clarified a novel pop clamor identification plan to perceive the pinpoint pop commotions at the phonemic level. Which obviously delivers the breathing of the client while talking near the mouthpiece [7].

The creator Morgen, B. Recommended a recognizable proof and confirmation (ID\&V) innovation for the verification. It utilized the hierarchical information of clients [16].

The creator DanMillera et al. proposed a Multifactor validation instrument. It manufactures a standard correspondence methodology for biometric speaker recognizable proof and confirmation [17].

The maker DanMillera et al. proposed a Multifactor approval instrument. It fabricates a standard correspondence system for biometric speaker unmistakable verification and affirmation [17].

The creator GauravVerma et al. proposed a face biometric system dependent on optical change. The change of a biometric done through the stage recovery calculation. It builds up the two-factor confirmation system [18].

The creator Keane, S. Et al. certified voice acknowledgment systems. It receives the voice biometric adjusted to the general population. This examination concentrated on the two nations new Zealand and Australia [19].
The creator Elizabeth A. Simpson et al. investigated a face location framework to empower the nursery-raised newborn child macaques. It takes consideration regarding the appearances in 10-things of an exhibit. The strong face location framework concentrated on nonface pictures [8]. The creator Anil K. Jain et al. Proposed a fire get print acknowledgment method, which will take from the youthful age of the kid. It gives higher exactness of the hehe fingerprints taken from the half-year-old kid [9].

The creator Linghan Zhang et al. Depicted a VoiceLive to distinguish the liveness of voice validated on cell phones. time-distinction of-appearance (TDoA) caught an arrangement of two-mouthpiece on a telephone. It requires extra equipment yet a two-channel stereo chronicle [12].

The creator Jan Chorowski et al. Proposed a TIMIT phoneme acknowledgment task. It determined by the phoneme blunder rate (PER). The exhibitions dependent on machine-interpretation, penmanship union, and picture catch age [13].

The creator Michel Vacher et al. depicted a voice-controlled multi-room savvy home ASR and speaker recognizable proof frameworks. The significant effect of the framework concentrated on the separation while accepting voice. Herewith this procedure chooses the best channel works powerfully. The conditions work dependent on natural conditions [14].

The creator SasanAdibi et al. suggested a low overhead voice confirmation conspire. The highlights assumed control over the adjustment and scaling of the voice recurrence sounds. Voice confirmation takes a significant separation while getting [15].

The creator Alexander De Luca et al. Proposed an ACM order to concentrated on biometric confirmation courses in cell phones. There are various components utilized in a cell phone to open. Security and trust issues are the main considerations here [1].

The creator Mohammad Sabzinejad Farash depicted a validation over the web. Ordinarily, the session inception convention (SIP) utilized for confirmation from essentials. To defeat the downside the creator improved another method called elliptic bend cryptography (ECC) with that SIP [2].

The maker Kimmo Halunen et al. portrayed a customer approval instrument at five raised levels features. Which performed in the gadget-free world. A single approval framework releases all of the necessities. However, this blend of the approval factor enables to achieve all of the essentials [20].

The maker Vennila, G et al. proposed a Dynamic Voice Spammer Detection model(DVSDM) considering the Hidden Markov Model(HMM). It perceives the voice spammers in preceding the individual being referred to. It achieves a True Positive Rate(TPR) and False Positive Rate(FPR) [21].

\section{TWO LEVEL VOICE AUTHENTICATION PROCESS}

In this examination, the mystery key would be produced at first relying upon the arbitrary qualities inputted by the client.

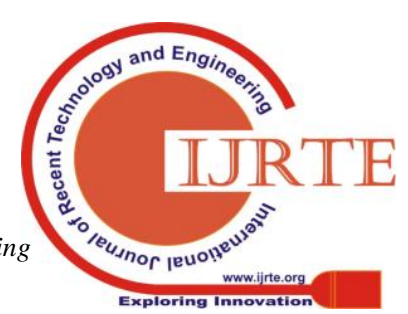


Encryption of the client's compacted voice sign will be executed dependent on the mystery key and the voiceprint will be acquired on the server-side. Further, the voiceprint got will be put to use for the confirmation procedure. This will give effective aversion to the clients from Compromisation assaults performed by within assailants.

\subsection{RANDOM VALUE GENERATION PHASE}

This phase comprises of random numbers generated by each node. Then for further authentication, these random numbers will be shared with the cluster head. A sequence of symbols and numbers are obtained from the random number generator (RNG) device which is effective in comparison with the other devices. Genuinely random numbers are outputted by True hardware random-number generators (HRNG) or pseudo-random number generators (PRNG) in which the output just looks random is the random number generators. Though these are deterministic and are reproducible if the PRNG state is known. The sequence of random numbers is obtained from the mathematical formulas obtained from the algorithm, Pseudo-Random Number Generator (PRNG). The sequence numbers are generated by PRNGs by approximation of properties of random numbers. The arbitrary starting state is where PRNG starts using a seed state. If This stage includes irregular numbers created by every hub. At that point for further verification, these irregular numbers will be imparted to the group head. A succession of images and numbers is acquired from the irregular number generator (RNG) gadget which is compelling in correlation with different gadgets. Really arbitrary numbers are yielded by True equipment irregular number generators (HRNG) or pseudo-irregular number generators (PRNG) in which the yield just looks irregular is the arbitrary number generators. Despite the way that these are deterministic and are reproducible if the PRNG state is known. The course of action of optional numbers is acquired from the numerical plans got from the figuring, Pseudo-Random Number Generator (PRNG). The course of action numbers is conveyed by PRNGs by the hypothesis of properties of optional numbers. The discretionary beginning state is the spot PRNG starts utilizing a seed state. If the starting stage appeared in the course of action, by then, various numbers are being passed on in a limited capacity to center time and they are reproducible later. In this manner, they are viewed as reasonable and deterministic.

The headway in PCs has prompted distinguishing the requirement for presentation of haphazardness by software engineers into a PC program. In spite of the fact that this may appear to be amazing, it is a provoking undertaking to make the PC to perform something by chance as it aimlessly adheres to inputted guidance and is totally unsurprising. The age of genuinely arbitrary numbers by PC, a deterministic gadget is incredibly impractical, so the PRNG method is utilized to create irregular numbers utilizing PC.

beginning stage distinguished in the grouping, at that point numerous numbers are being created in a limited capacity to focus time and they are reproducible later. Along these lines, they are seen as productive and deterministic.

The progression in PCs has prompted recognizing the requirement for the presentation of haphazardness by developers into a PC program. Despite the fact that this may appear to be astonishing, it is a moving assignment to make the PC to perform something by chance as it indiscriminately adheres to inputted guidance and is totally unsurprising. The age of genuinely arbitrary numbers by PC, a deterministic gadget is incredibly impractical, so the PRNG system is utilized to produce irregular numbers utilizing a PC.

Direct Congruential Generator is seen as the most seasoned and most regular calculation for the age of pseudo-irregular numbers. This is depicted by methods for repeat relationships.

$$
X_{n+1}=\left(a X_{n}+c\right) \bmod
$$

Where the sequence of pseudo-random values is represented by $\mathrm{X}$.

$\mathrm{m}$ is a modulus and it ranges like $0<\mathrm{m}$

$\mathrm{a}$ is a multiplier and it ranges between $0<\mathrm{a}<\mathrm{m}$

$\mathrm{c}$ is an increment and it ranges between $0<=\mathrm{c}<\mathrm{m}$

$\mathrm{x}_{0}$ is started value of seed and it ranges between $0<=\mathrm{x}_{0}<\mathrm{m}$

Number constants, past irregular whole numbers, and whole number modulus are utilized to produce the following arbitrary number. To begin, the calculation requires an underlying Seed. Modulo math is being performed to get the presence of irregularity. The acquired arbitrary numbers are then mutual with the server to perform further activities.

\subsection{SECRET KEY GENERATION AND SHARING PHASE}

Give X a chance to be either an arbitrary worth or topsy-turvy key utilized to include an endorsed awry key pair creating the calculation. It is spoken to by utilizing a string with the accompanying articulation:

$$
\mathrm{X}=\mathrm{U} \oplus \mathrm{V}
$$

Where the ideal length of the bitstream is spoken to by - U and yield of endorsed PRNG produces this, fit for offering help to the ideal security quality all together for the insurance of the objective information,

The bit string is spoken to by,

- V speaks to and its length is equivalent to the length of $U$ and it is structured so that it is free of the estimation of $U$

Security quality upheld by this utilization and the calculation alongside $\mathrm{X}$ will be utilized to decide bit length as well as the least security quality required that ought to be given by this procedure.

$\mathrm{X}$ will be utilized to decide the bit length or potentially least security quality required that ought to be given by this procedure. The determination of $\mathrm{V}$ isn't having any limitation, the technique expects that the procedure utilized in the choice of $U$ yields most (if not the majority) of the required entropy. Understanding by methods for computational and factual methods is performed on the freedom prerequisites on $U$ and $\mathrm{V}$, implies the calculation of $\mathrm{V}$ isn't reliant on $\mathrm{U}$, the calculation of $\mathrm{U}$ isn't subject to $\mathrm{V}$, and by having learning theta one qualities (V or U)

will deliver no data which is utilized to pick up knowledge into different qualities. On suspicion of $U$ gives a yield of endorsed PRNG, autonomously chose $\mathrm{V}$ esteems to think about the accompanying models.

1. $\mathrm{V}$ is a steady (it is freely chosen from $\mathrm{U}$ esteem $)$. ( $\mathrm{K}=\mathrm{U}$, if

Published By: and Engin

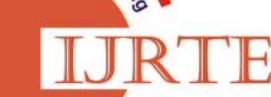
$\mathbb{I J} \mathbb{R} T E$ s Publication 


\section{Patterned Endorsement Framework for Defense Sector using Two Level Voice Authentication Process}

$\mathrm{V}$ is a string of twofold zeroes. It compares to the yield of an affirmed PRNG.)

2. key-induction technique is utilized to discover the estimation of $\mathrm{V}$ and different information sources are being autonomous of $\mathrm{U}$;

3. In another cryptographic module, keyV is created freely. An affirmed key wrapping calculation is utilized for ensuring $\mathrm{V}$ or is moved. After the gathering, insurance on $\mathrm{V}$ is expelled in the key age module which created $U$ past consolidating $U$ with $\mathrm{V}$.

4. By utilizing another piece string $\left(\mathrm{V}^{\prime}\right), \mathrm{V}$ is created by utilizing the endorsed capacity and (whenever required) consolidate the result to fitting length before joining it with $U$. That is,

$$
\mathrm{V}=\mathrm{T}\left(\mathrm{H}\left(\mathrm{V}^{\prime}\right), \mathrm{k}\right)
$$

where $\mathrm{T}(\mathrm{x}, \mathrm{k})$ speaks to the truncation of bit string $\mathrm{x}$ to its $\mathrm{k}$ furthest left bits, where the length of $\mathrm{U}$ is given by $\mathrm{k}$. The bit string $\mathrm{V}^{\prime}$ may go about as a) consistent; b) key is gotten from the common mystery; or c) a key that was I) being created freely by another module, ii) moved to utilize key wrapping calculation or an affirmed key vehicle plot and iii)the security was being evacuated upon receipt.

\subsection{VOICE SIGNAL COMPRESSION}

Sign planning incorporates a known and by and largely used methodology called Discrete Wavelet Transform (DWT). The crumbling of the sign is being performed by this strategy over the low pass and high pass channel. To bring different sorts of low pass and high pass channels, different coefficients are worked for immense choices between different elucidations and scales. for instance Symlets, Debauchies, Coif lets. The state of the channel for one level of rot is given as seeks after.

$$
\begin{aligned}
\mathrm{A}[\mathrm{k}]= & \sum \mathrm{x}[\mathrm{n}] \times \mathrm{h}(2 \mathrm{k}-\mathrm{n}) \\
& \mathrm{D}[\mathrm{k}]=\sum \mathrm{x}[\mathrm{n}] \times \mathrm{g}(2 \mathrm{k}-\mathrm{n})
\end{aligned}
$$

Where $A[k]$ speaks to the yield of low pass channel (guess), $\mathrm{h}[\mathrm{n}]$ speaks to the half band of low pass channel, $\mathrm{g}[\mathrm{n}]$ speaks to the half band of high pass channel given by and discrete type of the first sign is spoken to by $x[n]$. The deterioration of sign is performed by DWT where another sign is shaped at every estimation, at the following level. Electro Cardio Gram (ECG) signal decay [1] includes a significant advance of determination of wavelet work and the chose sign ought to be close enough to the broke down the sign. This technique contains Symlet 7 (sym 7), a picked capacity and this capacity yields the powerful outcome in correlation with other proposed strategies.

Disposing of information is allowed by the hard limit as pursues by contrasting and edge coefficient and it is spoken to by $\varphi$ and result vector of wavelet decay is given by $\mathrm{C}[\mathrm{n}]$ : If $|\mathrm{C}[\mathrm{n}]|<\varphi$, then $\mathrm{C}[\mathrm{n}]=0$

Else $\mathrm{C}[\mathrm{n}]$ is free of thresholding;

The decrease of clamors accessible in the subsequent guess is performed by the thresholding step and distributes similar qualities to all commotions. Versatile thresholding has been proposed in this paper as pursues

$\varphi=\alpha \times \max _{\mathrm{n} 1 \rightarrow \mathrm{n} 2}|\mathrm{C}[\mathrm{n}]|$

where $\alpha$ speaks to the parameter of thresholding, $\mathrm{n} 1$ to $\mathrm{n} 2$ speaks to the length in detail. The straightforward thresholding of vector $\mathrm{C}$ is done in the proposed technique, wherein parameter $\alpha$ is a chief factor of thresholding.

\subsection{ACOUSTIC SIGNAL ENCRYPTION}

Our cryptosystem incorporates the talk signal as segments by techniques for using three puzzle keys. Indirect move (inline and segment) is used by the system of the stage which is being handled from encryption key bits and the encryption structure uses substitution on different characteristics for the departure of sign lucidness by techniques for DST or DCT. By at that point, the data talk sign is separated and reshaped into fixed-size squares to such an extent, that the size of the square depends upon a mystery key. Coming up next are the techniques for encryption estimation.

\section{Algorithm 1 ENCRYPTION ALGORITHM}

Step 1: The 2-D blocks are obtained by means of Framing and reshaping.

Step 2: Row and column-wise Circular shifts

1 st Round

Step 3: Generate main key K1

Step 4: Using main key K1 perform permutation

Step 5: Generate Mask M1

Step 6: then add mask M1

2 nd Round

Step 7: Apply Discrete Sine Transform (DST) or Discrete Cosine Transform (DCT)

Step 8: Generate second key K2

Step 9: Using second key K2 perform Permutation

Step 10: Generate Mask M2

Step 11: Add Mask M2

3 rd Round

Step 12: Apply Inverse Discrete Sine Transform (IDST) or Inverse Discrete Cosine Transform (IDCT)

Step 13: Third key K3 is generated

Step 14: using key K3 perform permutation

Step 15: Then reshaping into a 1-D format

Encryption calculation incorporates tested discourse signal and recorded a discrete structure with the abundancy that lies between - 1 and 1 as its initial step. At that point, the arrangement of tests is being joined as square squares in which the width approaches the length of the mystery key.

\subsection{DECRYPTION OF RECEIVED VOICE SIGNALS}

The opposite activity system is applied to the scrambled messages by the unscrambling calculation to recover the first message. The decoding of encoded discourse is executed as pursues:

\section{Algorithm 2 DECRYPTION ALGORITHM}

-Step 1: Generate three secret keys K1, K2, and K3

Step 2: Use framing and re-shaping to construct 2-D block

Step 3: Use third key K3, to perform inverse permutation

Step 4: Apply Discrete Sine Transform (DST) or Discrete Cosine Transform (DCT)

Step 5: Generate mask M2

Step 6: Mask M2 is subtracted

Step 7: use second key K2 to perform inverse permutation

Step 8: Apply inverse Discrete Sine Transform (IDCT) or inverse Discrete Cosine Transform (IDST)

Step 9: Generate mask M1

Step 10: Mask M1 is subtracted

Step 11: Use the main key K1 
to perform inverse permutation

Step 12: Perform inverse Circular shifts in means of row and column

Step 13:1-D format is obtained by reshaping

\subsection{SYNCHRONY BASED FEATURE EXTRACTION}

We have broken down a few strategies for the extraction of synchrony data for discourse acknowledgment applications. The extraction of synchrony endeavors in this handling in such a way which mirrors the first sign's recurrence content as opposed to simply extricating the middle recurrence of every examination channel. The result from each channel of the sound-related model is gone at first during that time bandpass channel which has indistinguishable recurrence reactions from that of the sound-related channel for that channel to limit the consonant contortion which is presented by methods for nonlinearities in the fringe sound-related preparing.

The calculation of the result of the bandpass channels from the brief span Fourier change is separated, and the acquired recurrence reactions have arrived at the midpoint of crosswise over channels. The high-goals ghastly portrayal is created by this in low frequencies, for which the sound-related nerve is tuned to deliver the contribution of up to $2.2 \mathrm{kHz}$ and furthermore in fringe handling, it includes the impacts of all nonlinearities. Narrowband spectrograms(reflects the pitch of moving toward sign) ordinarily contain the level striations and its ejection is performed by techniques for applying the discrete-cosine change (DCT) on the repeat responses by strategies for applying the brief time span "lifter" for turn around change. By then, it returns to the repeat zone by using speak DCT.

The discourse acknowledgment includes highlights that are created at low frequencies by methods for blending the synchrony yields with the mean rate results at higher frequencies.

The synchrony yields from the start raise as the direct capacity of recurrence at the underlying stage and afterward they are wrapped along the recurrence pivot with the end goal that the mean rates are wrapped along the recurrence hub which in term relates to the inside recurrence called nonlinear reliance on recurrence for building up the mean rate yields.

The result parts from the recurrence wrapped synchrony lie between the estimate of 0 and $2.5 \mathrm{kHz}$ and the yield from the mean rate remains in the guess of 2.2 to $8 \mathrm{kHz}$ which are protected for future preparing. The yields acquired are exposed to last DCT from which the arrangement of coefficients is added which are like that of cepstral coefficients.

The initial 8 DCT coefficients are utilized by the present execution that is being gotten from the synchrony yield. The mean rate yield is the place the initial 5 DCT is inferred. Delta and delta-delta coefficients are gotten along these lines as is regularly cultivated for the Carnegie Mellon University (CMU) SPHINX system.

\subsection{SPEECH RECOGNITION USING IMPROVED ARTIFICIAL NEURAL NETWORK}

A Hybrid Particle Swarm Optimization-Artificial Neural Network (HPSO-ANN), is being executed in the proposed approach to manage rushed out the result of talk insistence of the entire structure. Learning and testing are the two major strolls in the HSPO-ANN classifier. The structure of the
HPSO-ANN classifier close by the PSO estimation is delineated in Figure 1.

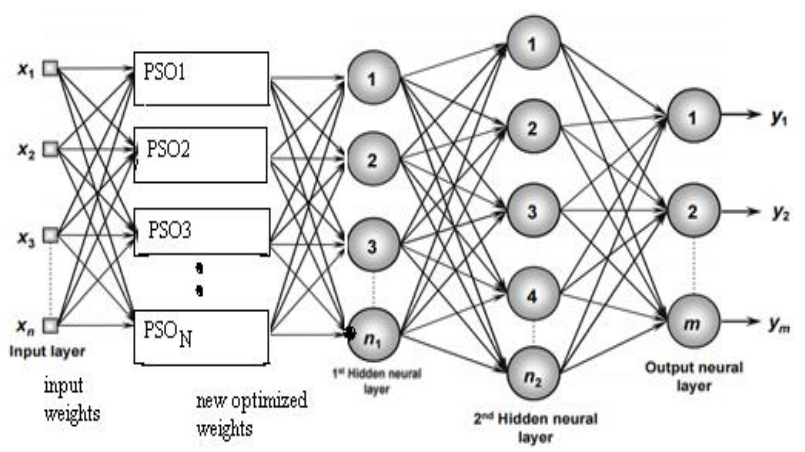

Figure. 1. Improved Artificial Neural Network (IANN)

The ideal size of the discourse datasets likely the quantity of concealed units in center layers, complete depending on layers, preparing calculation and number of units in information and yield layers by methods for precision on test set were authorized and would execute the boisterous preparing set for accomplishing the great discourse upgrade. The learning focuses on lessening the cost capacity regarding parameters (amount) contingent upon the blunder signal ei(t) with the end goal that the objective reaction is accomplished by the genuine reaction. The ongoing work incorporates the data on the HPSO-ANN classifier.

\section{RESULTS AND DISCUSSION}

The proposed TLVAS assessment is performed by methods for utilizing the tangle lab reenactment condition. The voiceprint is being assessed against each other for the distinguishing proof of better execution.

\subsection{SENSITIVITY}

Affectability is the assessment of rate including genuine positive for characterizing the real voice as real. The meaning of affectability is as per the following:

Sensitivity $=\frac{T_{p}}{T_{p}+F_{n}}$

Where T_pis the certified voice which is perceived accurately as the verified voice.F_p Represents the interloper voice that is mistakenly validated as gatecrasher's voice. $\mathrm{F} \_\mathrm{n}$, the interloper's voice is erroneously recognized as the validated voice. The gatecrasher's voice $T_{-} n$ is perceived accurately as the interloper's voice.

\subsection{PRECISION}

Accuracy can be characterized as the extent of genuine positives thought about against the genuine positives just as false positives result from the voice signal. The definition is as per the following:

$$
\text { Precision }=\frac{T_{p}}{T_{p}+F_{p}}
$$




\subsection{ACCURACY}

The general rightness of the model is characterized in the terms of precision and is being determined as pursues: the total of genuine order parameters $\left(\mathrm{T} \_\mathrm{p}+\mathrm{T} \_\mathrm{n}\right)$ to the absolute number of characterization parameters $\left(T \_p+T \_n+F \_p+F \_n\right)$.

Accuracy $=\frac{T_{p}+T_{n}}{T_{p}+T_{n}+F_{p}+F_{n}}$

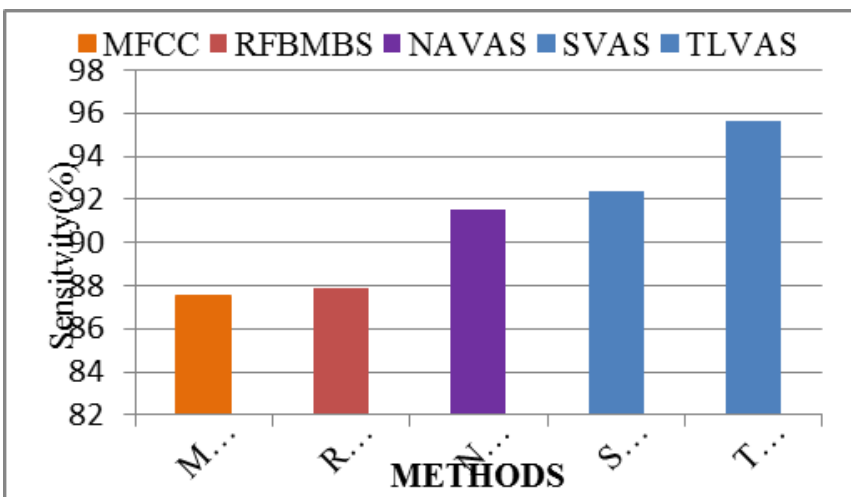

Figure 2. Performance Evaluation of Sensitivity with Different Methods

The appraisal of affectability is performed and it is found that the proposed way of thinking is viewed as better than that of the current framework.

The evaluation result portrays as seeks after $87.5 \%$ is for MFCC, Retina, and Fingerprint Based Multi - Biometric System (RFBMBS) procedure metric incorporates $87.9 \%$, $91.5 \%$ is of NAVAS, SVAS is about $92.4 \%$ and TLVAS has $95.6 \%$. In a relationship with various procedures, figure 2 depicts better affectability.

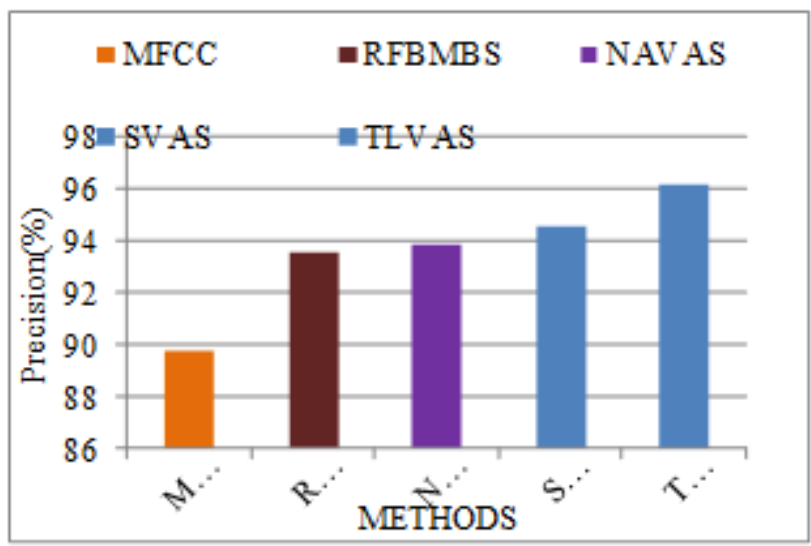

Figure 3. Performance Evaluation of Precision with Different Methods

The proposed approach in this exactness assessment is seen as superior to that of existing strategies. The MFCC involves $89.7 \%, 93.5 \%$ is for RFBMBS, NAVAS comprises of $93.8 \%$. $94.6 \%$ is for SVAS and the TLVAS has $96.2 \%$. The better execution by methods for affectability is portrayed in figure 3 , in correlation with different strategies.

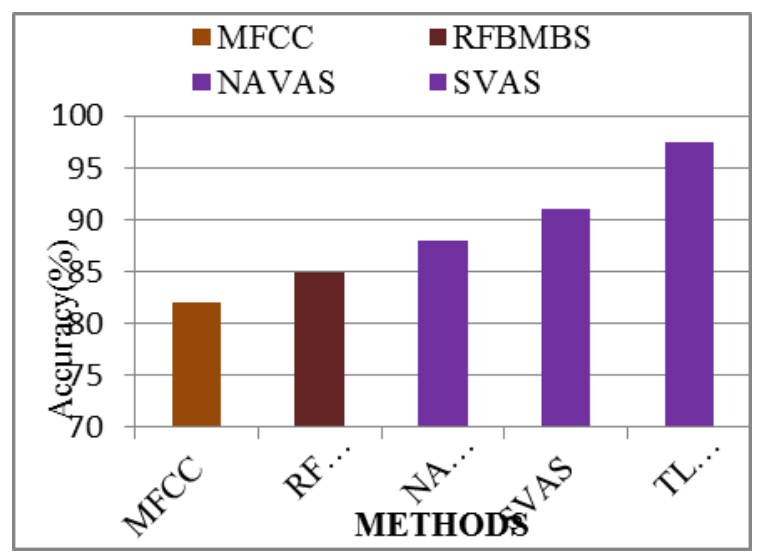

Figure 5: Performance Evaluation of Accuracy with Different Methods

Exactness assessment of the proposed strategy additionally beats than that of the current technique. MFCC technique involves $82.00 \%, 85.00 \%$ is of RFBMBS, NAVAS is $88 \%$, $91 \%$ is contained SVAS and TLVAS has a level of $97.5 \%$. Figure 5 delineates the better affectability investigation in correlation with that of different systems.

\section{CONCLUSION}

This examination work includes the age of the mystery key contingent upon the arbitrary information sources put together by the client. The encryption of the voice sign is being performed by utilizing the mystery key to get the voiceprint on the server-side. The voice print acquired would be additionally utilized with the end goal of the confirmation procedure. The productive avoidance is given from the assaults held by within aggressors, by this usage work. The result acquired is put to the presentation investigation which is being done in the tangle lab and the correlation is performed by methods for expansion in security level.

\section{REFERENCE}

1. De Luca, A., Hang, A., Von Zezschwitz, E., \& Hussmann, H. (2015, April). I feel like I'm taking selfies all day!: Towards understanding biometric authentication on smartphones. In Proceedings of the 33rd Annual ACM Conference on Human Factors in Computing Systems (pp. 1411-1414). ACM.

2. Farash, M. S. (2017). An improved password-based authentication scheme for session initiation protocol using smart cards without a verification table. International Journal of Communication Systems, 30(1), e2879.

3. Ikeno, A., Shimada, M., Hatanaka, K., Nishijima, T., Kataoka, F., Tonegawa, H., \&Umeyama, N. (2018). U.S. Patent Application No. $15 / 692,633$

4. Perotti, E. K. (2018). U.S. Patent Application No. 15/439,588.

5. Hossain, M. S., \& Muhammad, G. (2015). Cloud-assisted speech and face recognition framework for health monitoring. Mobile Networks and Applications, 20(3), 391-399.

6. Beckley, J. D., Aggarwal, P., \&Balasubramanyam, S. (2018). U.S Patent No. 9,881,616. Washington, DC: U.S. Patent and Trademark Office.

7. Wang, Q., Lin, X., Zhou, M., Chen, Y., Wang, C., Li, Q., \& Luo, X. (2019, April). VoicePop: A Pop Noise based Anti-spoofing System for Voice Authentication on Smartphones. In IEEE INFOCOM 2019-IEEE Conference on Computer Communications (pp. 2062-2070). IEEE.

8. Fagan, J. F. (2017). The origins of facial pattern recognition. In Psychological development from infancy (pp. 83-113). Routledge.

9. Jain, A. K., Arora, S. S., Cao, K., Best-Rowden, L., \& Bhatnagar, A. (2016). Fingerprint recognition of young children. IEEE Transactions on Information Forensics and Security, 12(7), 1501-1514.

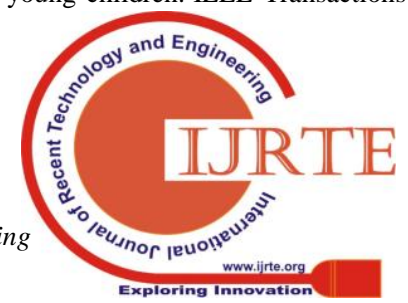


10. Talhami, H. E., Malegaonkar, A. S., Malegaonkar, R. A., \& Summerfield, C. D. (2016). U.S. Patent No. 9,424,837. Washington, DC: U.S. Patent and Trademark Office.

11. Nagel, R. H., Jones, P., Wener, C., \&Readick, J. (2017). U.S. Patent No. 9,788,199. Washington, DC: U.S. Patent and Trademark Office.

12. Zhang, L., Tan, S., Yang, J., \& Chen, Y. (2016, October). Voicelive: A phoneme localization-based liveness detection for voice authentication on smartphones. In Proceedings of the 2016 ACM SIGSAC Conference on Computer and Communications Security (pp. 1080-1091). ACM.

13. Chorowski, J. K., Bahdanau, D., Serdyuk, D., Cho, K., \&Bengio, Y. (2015). Attention-based models for speech recognition. In Advances in neural information processing systems (pp. 577-585).

14. Vacher, M., Lecouteux, B., Romero, J. S., Ajili, M., Portet, F., \&Rossato, S. (2015, October). Speech and speaker recognition for home automation: Preliminary results. In 2015 International Conference on Speech Technology and Human-Computer Dialogue (SpeD) (pp. 1-10). IEEE.

15. Adibi, S. (2014). A low overhead scaled equalized harmonic-based voice authentication system. Telematics and informatics, 31(1), 137-152.

16. Morgen, B. (2012). Voice biometrics for customer authentication. Biometric Technology Today, 2012(2), 8-11.

17. Miller, D., \& Fauve, B. (2012). Mobile e-commerce to drive voice-based authentication. Biometric Technology Today, 2012(2), 5-8.

18. Verma, G., Liao, M., Lu, D., He, W., \& Peng, X. (2019). A novel optical two-factor face authentication scheme. Optics and Lasers in Engineering, 123, 28-36.

19. Keane, S. (2010). Banking on voice for large scale remote authentication. Biometric Technology Today, 2010(8), 8-10.

20. Halunen, K., Häikiö, J., \&Vallivaara, V. (2017). Evaluation of user authentication methods in the gadget-free world. Pervasive and Mobile Computing, 40, 220-241.

21. Vennila, G., Manikandan, M. S. K., \& Suresh, M. N. (2018). Dynamic voice spammers detection using Hidden Markov Model for Voice over Internet Protocol network. Computers \& Security, 73, 1-16.

\section{AUTHORS PROFILE}

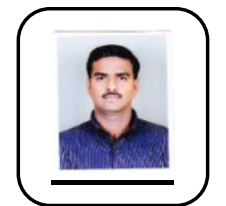

M. Kathiresh is a Research Scholar at Erode Arts and Science College, Erode. He received his B.Sc Degree in Mathematics from Bharathiar University.MCA Degree at Bharathiar University and M.Phil Degree at Karpagam University. He has more than 9 years of teaching Experience and he published 5 Articles. His research interest includes Network Security, Compiler Design, and Software Engineering. He has finished one UGC sponsored Minor Research Project.

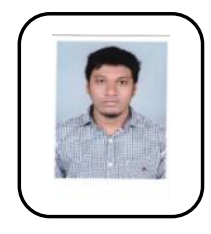

N. Shunmuganathan is a Research Scholar at Erode Arts and Science College, Erode. He received his BCA Degree from Bharathiar University. M.sc IT from SRM University and M.Phil Degree in Bharathiar University. His research interest includes Digital Image Processing, Software Engineering. He has more than 3.5 years in IT Sector.

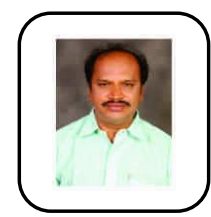

Dr.R.Sankarasubramanian is working as an Associate Professor in the Department of Computer Science, Erode Arts and Science College, Erode. He received his $\mathrm{Ph} . \mathrm{D}$. degree from Bharathiar University. He has more than 27 years of teaching, 19 years of Research experience. His research interests include Network Security, Software Testing. 